\title{
Continuous Recording of Plasma Sodium Concentration and Blood Volume in Awake Rats
}

\author{
Hiroshi Nose, Eizo Sugimoto, Taketoshi Morimoto, \\ Seiji UsuI,* and Takashi Aomi* \\ Department of Physiology, Kyoto Prefectural University of Medicine, \\ Kamigyo-ku, Kyoto, 602 Japan \\ * Horiba Ltd., Minami-ku, Kyoto, 601 Japan
}

\begin{abstract}
Summary A new continuous measuring system for blood sodium concentration and blood volume in conscious rats is described. A small extracorporeal circulation was made and the blood was passed through a sodium sensitive glass electrode and a $\gamma$-counter. Applying the system to conscious rats, the effect - of various salinities of drinking water was detected from the change in blood sodium concentration.
\end{abstract}

Key words: continuous recording, sodium sensitive glass electrode, conscious rat.

Sodium concentration of plasma ([Na]) and blood volume (BV) are known to modify drinking behavior (FITZsIMONS, 1979). To analyze drinking behavior, it is necessary to determine [Na] and BV without disturbing BV by blood samplings especially in small animals such as rats. Therefore, an attempt was made to construct a new system to measure plasma [Na] and BV simultaneously and continuously on conscious rats without blood samplings. The system utilizes a commercially available electrolytes analyzer with glass electrodes to measure $[\mathrm{Na}]$ as the blood is pumped through the electrode.

At least 5 days prior to the experiment, rats were splenectomized and cannulae were inserted into the descending aorta (a polyethylene tubing PE 50 fused to PE 10, Clay-Adams, NJ) and the jugular vein (PE 60). The other ends of cannulae were exteriorized through an interscapular incision, threaded through a flanged and coiled stainless steel spring, and connected to a cannula swivel (Muromachi Kikai, Tokyo). The cannulae were flushed daily with $0.1 \mathrm{ml}$ of heparinized saline $(1,000 \mathrm{U} / \mathrm{ml})$. The cannulated rat could move freely within a cage $(20 \times 20 \mathrm{~cm})$ and had free access to food and water. On the study day, the cannulae were connected with a pump (minipulse pump, Gilson, Villiers-le-bel, France) to the system for

Received for publication February 3, 1986 
continuous measurements of $[\mathrm{Na}]$ and $\mathrm{BV}$. The $[\mathrm{Na}]$ was determined by a sodium sensitive glass electrode and $\mathrm{BV}$ was measured by a dilution method using ${ }^{51} \mathrm{Cr}$ labeled erythrocytes. Details of the cannulation procedure and the continuous BV determination have been published elsewhere (NosE et al., 1986).

Measurement of [Na] was made using a sodium sensitive glass electrode (1411A), a reference electrode (2431A) mounted on a temperature control unit of an electrolyte analyzer (SERA-230, Horiba, Kyoto). In the commercially available analyzer, a flow junction of $\mathrm{KCl}$ solution is used to obtain a stable junction potential. However, in our system, the liquid junction was replaced by $3.3 \mathrm{M} \mathrm{KCl}$ agar bridge to minimize contamination of blood with $\mathrm{KCl}$. The system is shown diagramatically in Fig. 1a. The junction potential was most stable when the agar bridge was prepared with $5 \%$ agar powder, $3.3 \mathrm{M} \mathrm{KCl}, 140 \mathrm{mM} \mathrm{NaCl}$ adjusting $\mathrm{pH}$ to about 7.4 with Tris- $\mathrm{HCl}$ buffer solution. The electromotive force (emf) of the glass electrode was read with a high input-impedance amplifier (HC-100, Horiba, Kyoto); the output was recorded with a pen recorder (056, Hitachi, Tokyo) and a desk top computer (M223, Sord, Tokyo) after analog-digital conversion. The electrodes and tubings connecting blood sample with the electrode system were maintained at a constant temperature adjusting the temperature control unit to $37^{\circ} \mathrm{C}$.

The accuracy of the system was determined using human serum samples and buffer solutions, and the relationship between serum sodium concentrations or Tris buffer solutions as determined with a flame photometer (480, Corning, Halsted, England) and the emf of the sodium glass electrode is depicted in Fig. 1b. As shown in the figure, a high correlation was observed and the $95 \%$ confidence limit of the regression line was $0.3 \mathrm{mV}$ which is equivalent to about $1.5 \mathrm{meq} / l$ of sodium concentration within this range. The sample $\mathrm{pH}$ ranged from 7.2 to 7.5 , potassium concentration was from 3.5 to $4.7 \mathrm{meq} / l$, and protein concentration from 6.7 to $7.4 \mathrm{~g} / \mathrm{dl}$. These deviations of emf include the effect of interfering ions as reported by Yoshimura et al. (1962) and JoHnson et al. (1985). The effect of erythrocytes on the emf was also checked using whole blood with 42 to $51 \%$ of hematocrit, and deviations in emf reading were within $0.1 \mathrm{mV}$.

For animal experiments, the electrode was calibrated with standard $\mathrm{NaCl}$ solutions $(130,140$, and $150 \mathrm{~mm})$ whose $\mathrm{pH}$ were adjusted within $7.2-7.5$ with Tris buffer. In addition, blood samples were obtained before and after the experiment. Plasma sodium concentration was determined by flame photometry and the value coincided with the value determined with the electrode within the accuracy of flame photometry. This also indicates that the effect of erythrocytes are negligible. Because the ionic strength was almost identical both in standard solutions and plasma, the value of sodium concentration was used in the analysis instead of sodium activity.

Fluid replacement experiments were performed on three rats. The rats were thermally dehydrated by about $6 \%$ as reported previously (NosE et al., 1985), and then they were provided with one of the following: tap water, 0.45 , or $0.9 \% \mathrm{NaCl}$ 

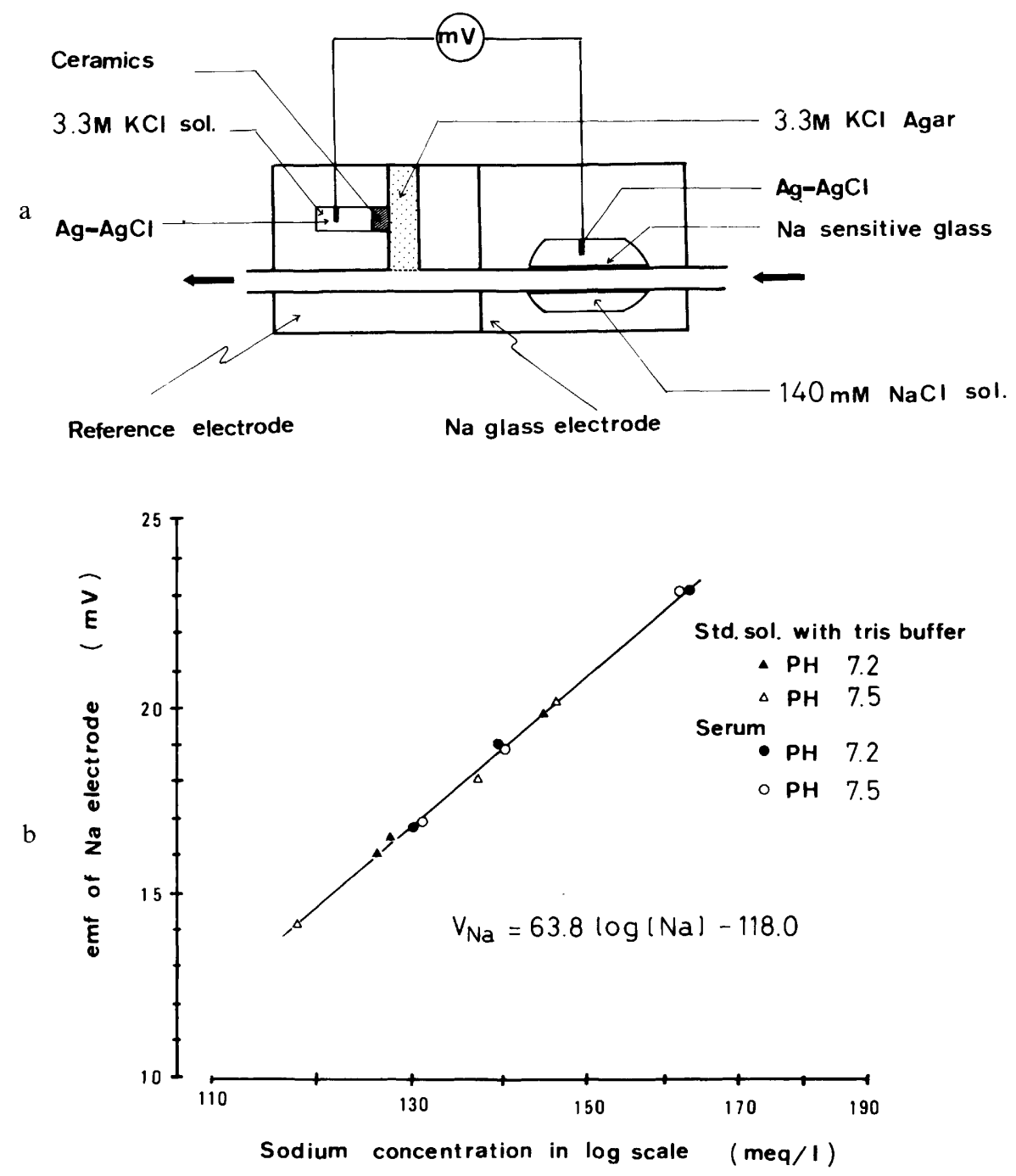

Fig. 1. Diagram showing electrode assembly (a); and sodium concentration-emf relationship obtained on standard solutions and serum samples (b). Correlation coefficient was 0.999 and $95 \%$ confidence limit was $0.255 \mathrm{mV}$.

solutions. The amount of fluid consumed was measured with a drop counter (Muromachi Kikai, Tokyo) and recoreded with the computer together with the change of BV and sodium concentration of circulating plasma. The results obtained on three rats are summarized in Fig. 2 in which the differences from the dehydration level are shown. The rats given $0.45 \% \mathrm{NaCl}$ solution consumed more fluid than those given tap water or $0.9 \% \mathrm{NaCl}$ solution. Higher increase in blood volume was observed in the rats given 0.45 or $0.9 \%$ saline solution while the rats given tap water 

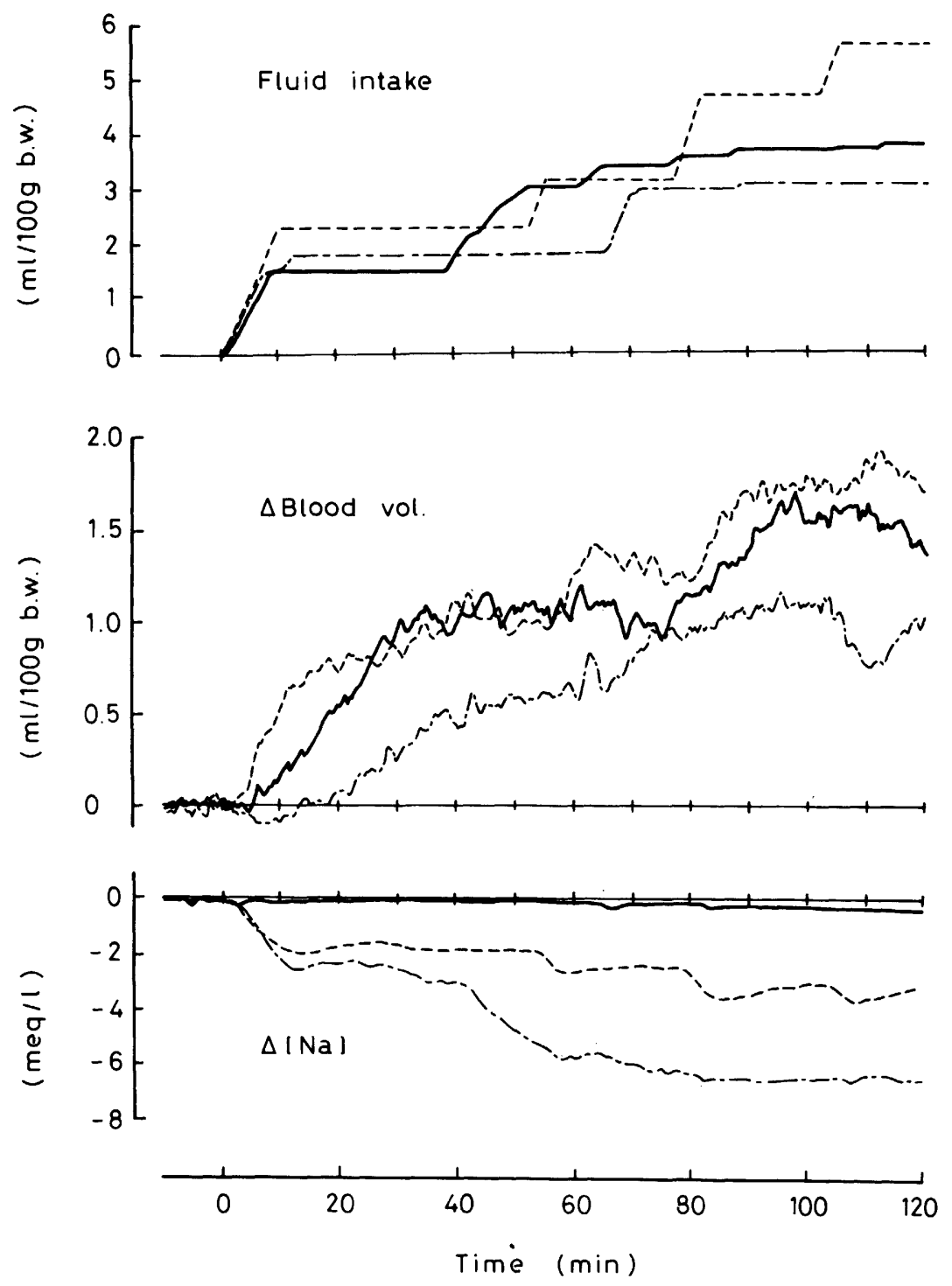

Fig. 2. Cumulative amount of fluid intake, changes in blood volume, and in blood sodium concentration of 3 rats. Rats were dehydrated and water was given ad libitum starting at $0 \mathrm{~min}$. Dashed and dotted lines indicate the changes observed in a rat given tap water, dashed lines represent the responses to drinking $0.45 \% \mathrm{NaCl}$ solution, and solid lines show the effects of drinking $0.9 \% \mathrm{NaCl}$ solution.

showed the least increase in blood volume. On the other hand, sodium concentration of the plasma showed a very clear-cut response. The rats given $0.9 \%$ saline showed no change in the plasma sodium concentration, while the rats given 
tap water showed a decrease of about $6 \mathrm{meq} / l$ within $60 \mathrm{~min}$. The rats given $0.45 \%$ $\mathrm{NaCl}$ solution showed a change equal to about one half of the response observed in the rats given tap water.

The results indicate that plasma $[\mathrm{Na}]$ reflects the salinity of drinking fluid. The technique described in this paper for continuous determination of sodium concentration with sodium sensitive glass electrode on bypassed blood is sensitive enough to detect small changes. The method is a promising tool for further analysis and understanding of drinking behavior under various conditions.

The authors are grateful to Dr. M. K. Yousef for critical reading of the manuscript and to Dr. Iyoda for advices on the chronic catheterization. This work was supported in part by Grant-in-Aid (60870007) for Developmental Scientific Research from the Ministry of Education, Science and Culture of Japan.

\section{REFERENCES}

Fitzsimons, J. T. (1979) The Physiology of Thirst and Sodium Appetite, Cambridge Univ. Press, Cambridge, pp. 128-222.

Johnson, D. C., Orlowitz, L., and Hitzig B. M. (1985) Differences between CSF and plasma $\mathrm{Na}^{+}$and $\mathrm{K}^{+}$activities and concentration. Am. J. Physiol., 248: R621-R626.

Nose, H., Morita, M., Yawata, T., and Morimoto, T. (1986) Continuous determination of blood volume on conscious rats during water and food intake. Jpn. J. Physiol., 36: 215-218.

Nose, H., Yawata, T., and Morimoto, T. (1985) Osmotic factors in restitution from thermal dehydration in rats. Am. J. Physiol., 249: R166-R171.

Yoshimura, H., HiraKawa, S., and Morimoto, T. (1962) Sodium sensitive glass electrode and its application to biological solutions. Seikagaku, 34: 154-158. 